\title{
Aprovechamiento de Cáscaras de Yuca y Ñame para el Tratamiento de Aguas Residuales Contaminadas con $\mathrm{Pb}$ (II)
}

\author{
Candelaria N. Tejada ${ }^{(1) \star}$, Zaida Montiel(1) y Diofanor Acevedo(2) \\ (1) Universidad de Cartagena, Facultad de Ingeniería, Programa de Ingeniería Química, Avenida el \\ Consulado, Calle 30 No. 48-152. Cartagena, Bolívar-Colombia (e-mail: ctejadat@unicartagena.edu.co) \\ (2) Universidad de Cartagena, Facultad de Ingeniería, Programa de Ingeniería de Alimentos, Grupo de \\ Investigación NUSCA, Avenida el Consulado, Calle 30 No. 48-152. Cartagena, Bolívar-Colombia
}

* autor a quien debe ser dirigida la correspondencia

Recibido May. 10, 2015; Aceptado Jul. 3, 2015; Versión final Ago. 22, 2015, Publicado Feb. 2016

\section{Resumen}

Se modificaron químicamente cáscaras de yuca y ñame con ácido cítrico y se evaluó su comportamiento para la adsorción de $\mathrm{Pb}$ (II). Los experimentos de adsorción se llevaron a cabo usando un tamaño de partícula en el rango de 0.5 a $1 \mathrm{~mm}$ y un $\mathrm{pH}$ de 6 . La determinación del metal remanente en solución fue medida mediante espectroscopia de absorción atómica. Se obtuvo una capacidad de adsorción de 52.34 y $98.36 \mathrm{mg} / \mathrm{g}$ para las cáscaras de yuca y ñame respectivamente. Además, se encontró que el modelo de Elovich y la isoterma de Langmuir y Freundlich ajustan adecuadamente los datos experimentales de adsorción. Se establece también que el enlace se da principalmente por fuerzas físicas, formándose solo una monocapa. Se concluye que los materiales en estudio modificados químicamente con ácido cítrico pueden ser usados para el tratamiento de aguas residuales, específicamente en la remoción de metales pesados como el plomo (II).

Palabras clave: cáscara de yuca; cáscara de ñame; materiales residuales; plomo; aguas residuales

\section{Utilization of Cassava and Yam Peels for the Treatment of Wastewater Contaminated with Pb (II)}

\begin{abstract}
Cassava and yam peels were chemically modified with citric acid and its performance for the adsorption of $\mathrm{Pb}$ (II) was evaluated. The adsorption tests were carried out using particle size between 0.5 and $1 \mathrm{~mm}$, and $\mathrm{pH}=6$. The determination of the remaining metal in solution was measured by atomic absorption spectroscopy. These adsorption capacities were of 52.34 and $98.36 \mathrm{mg} / \mathrm{g}$ for cassava and yam peels, respectively. In addition, it was found that Elovich's model and Langmuir's and Freundlich's isotherms adequately fit the adsorption experimental data. It is also established that the bond occurs mainly due to physical forces, forming a single monolayer. It is concluded that the materials under study, which were chemically modified with citric acid, can be used for wastewater treatment, specifically for the removing of heavy metals such as lead (II).
\end{abstract}

Keywords: cassava peel; yam peel; waste materials; lead; residual waters 


\section{INTRODUCCIÓN}

El incremento de la concentración de metales pesados en el agua se debe principalmente a la descarga de efluentes industriales, lo cual se ha convertido en el mayor problema de contaminación en todo el mundo, puesto que las concentraciones de metales en agua han excedido los límites permitidos. Entre los principales metales causantes de contaminación se encuentran: torio, cadmio, plomo, cromo, arsénico, mercurio, cobre y níquel; de entre estos el plomo puede afectar principalmente el sistema nervioso central, órganos como el riñón y el hígado, y el sistema reproductivo (Ghasemiet al., 2014). Es esencial remover estos metales antes de que puedan ingresar al ambiente, así procesos comunes para su remoción incluyen: precipitación química, intercambio iónico, adsorción, filtración por membranas, filtración electroquímica, etc, de estos la adsorción es considerado como el método más simple y eficiente (Shih-Weiet al., 2011).

Comparada con otras técnicas la adsorción puede ser considerada como favorable, ya que es altamente eficiente, incluso a bajas concentraciones del metal. Por lo tanto, el desarrollo de nuevos adsorbentes es una materia que ha cobrado gran importancia, no sólo por encontrar materiales eficientes, sino además económicos, tales como los residuos de algunos procesos industriales y actividades agrícolas (Ning-chuan y Xue-yi., 2012; Tejada et al., 2015). Teniendo en cuenta que en el departamento de Bolívar (Colombia) el cultivo de la yuca y el ñame están considerados entre los principales de su producción agrícola; según el ministerio de agricultura y desarrollo rural en el 2013, el departamento de Bolívar produce anualmente 139.355 toneladas/año de Ñame y 385.382 toneladas/año de yuca; siendo estos un renglón importante de la economía, lo que origina a su vez la producción de grandes toneladas de residuos, que actualmente sólo son usados como alimento para animales y en el peor de los casos son considerados desechos, sin ninguna aplicación. La literatura reporta mayor cantidad de trabajos de investigación con la yuca (Manihot esculenta), respecto al aprovechamiento de cascaras de ñame; para el caso de la yuca en el área de adsorción de metales se reportan trabajos en la remoción de cobre, cadmio, níquel, plomo, cromo, entre otros (Edoun y Kuitche, 2014; Kosasih et al., 2010; Brousse et al., 2012; Onwu et al., 2014). También la literatura reporta algunas aplicaciones del aprovechamiento de la cascara de ñame referidas a la remoción de tintas y diversos colorantes en solución (Owamah et al., 2013; Vinoth et al., 2010).

La adsorción es conocida como una operación en la cual los componentes de una corriente liquida se adsorben en un material sólido, el cual generalmente está formado por partículas pequeñas y muy porosas, dándose por lo general en forma de una monocapa sobre la superficie de los poros del material usado, se caracteriza por ser altamente selectiva, por depender de la naturaleza de quien se adsorbe y de quien adsorbe y del tratamiento al cual se someta el sólido (Shih-Wei et al., 2011; Yu et al., 2013). El origen de los adsorbentes puede ser variado, aunque han tomado mucha importancia los adsorbentes naturales que son económicos y en su mayoria obtenidos de residuos agroindustriales cuya habilidad para la adsorción de metales ha sido demostrada (Shih-Wei et al., 2011). Entre los bioadsorbentes destacados para la adsorción de $\mathrm{Pb}$ (II) se encuentra el alga roja, cáscaras de naranja y tuna guajira.

En otros estudios Vizcaíno y Fuentes (2015) estudiaron la influencia del pretratamiento y el empaquetamiento mediante ensayos tipo batch en la biosorción de $\mathrm{Cd}, \mathrm{Pb}$ y $\mathrm{Zn}$; en los que se emplearon soluciones de sodio y calcio. Se obtuvo como resultado una mayor capacidad de sorción de las algas modificadas con $\mathrm{NaOH} 0,1 \mathrm{~N}$ y de la naranja y la tuna con modificación sucesiva con $\mathrm{NaOH}$ y CaCl2 $0.2 \mathrm{M}$, y una afección poco significativa $(\leq 1 \%)$ del proceso de sorción al empacar la biomasa en placas planas de tul poliéster. La eficiencia de remoción se determinó mediante un reactor de flujo continuo de columna fija con un volumen líquido de $400 \mathrm{~mL}, 75 \mathrm{~g}$ de biomasa y tiempos de retención promedio de 1 y $2 \mathrm{~h}$. Los resultados mostraron una eficiencia similar de las tres biomasas para remover cadmio y plomo, con promedios superiores al 95\%, mientras que el zinc se removió con mejor eficiencia (62\%) al emplear tuna modificada como sorbente.

Por otro lado Anwar et al., (2010), estudiaron la adsorción de Pb (II) y Cd (II) sobre cáscaras de banana por lotes, la variables evaluadas fueron dosis de adsorbente, $\mathrm{pH}$, tiempo de contacto y la velocidad de agitación. Se emplearon las isotermas de Langmuir, Freundlich y Temkin para describir el equilibrio de adsorción. Los valores máximos de $\mathrm{Cd}$ (II) y $\mathrm{Pb}$ (II) adsorbido, según la evaluación de la isoterma de Langmuir, fueron $5,71 \mathrm{mg}$ y $2,18 \mathrm{mg}$ por gramo de polvo de cáscaras de banana, respectivamente; el estudio concluye que estos materiales son un material de desecho con un buen potencial como adsorbente para eliminar metales tóxicos en soluciones acuosas. Jun-Xia et al., (2013), evaluaron el uso de bagazo de caña modificada magnéticamente y encontraron que la capacidad de adsorción para $\mathrm{Pb}$ (II) y Cd (II) fueron 1,2 y $1,1 \mathrm{mmol} / \mathrm{g}$, respectivamente, de igual forma los modelos cinéticos de pseudo-primer y pseudo-segundo orden podrían predecir bien el proceso de adsorción. Se encontró que el $\mathrm{Pb}$ (II) podría ser adsorbido selectivamente por el sorbente magnético y este fue mayor o igual a 4:1 respecto al Cd (II). También se encontró que el modelo de Langmuir era adecuado para predecir la isoterma de sorción en el sistema binario. Alcanzada una remoción de $248,6 \mathrm{mg} / \mathrm{g}$ para el plomo; estos investigadores concluyeron que el sorbente modificado magnéticamente tenía un buen potencial en el tratamiento de aguas residuales con metales pesados. 
Maldonado et al., (2011) probaron la capacidad de adsorción del kikuyo pretratado por hidrolisis acida y básica, estudiando como afectaban las distintas variables en la adsorción, logrando determinar que la capacidad de adsorción máxima fue de $139,35 \mathrm{mg} / \mathrm{g}$ con una dosis de biosorbente de $0,06 \mathrm{~g}$ y un $\mathrm{pH}$ óptimo de 6. De otra parte, mediante el uso de aserrín se logró una máxima eficiencia de adsorción de plomo del $96,12 \%$, a $\mathrm{pH}$ de 7 , y un tiempo de contacto de 30 minutos, a $10 \mathrm{~g} / \mathrm{L}$ de adsorbente. Los datos de este estudio indicaron una buena correspondencia con las isotermas de Langmuir y Freundlich. (Ghasemi et al., 2014). Así, explorar la implementación de biomasas, para su uso en operaciones como la bioadsorción para el tratamiento de efluentes, es una medida útil para reconocer la aplicabilidad de los residuos agroindustriales. En el caso de la cáscara de la yuca y la cáscara de ñame, en la mayoría de las situaciones son desechadas, y dada su abundancia como desecho en la región caribe colombiana, llama la atención su aprovechamiento para la remoción de $\mathrm{Pb}(\mathrm{II})$, reduciendo de esta manera los desechos generados y transformándolos en subproductos aptos para tratar la contaminación en los cuerpos de agua.

\section{MATERIALES Y MÉTODOS}

\section{Preparación de la biomasa}

Los agentes químicos usados en el presente estudio fueron grado analítico marca Merck. Además para todos los análisis se utilizó agua desionizada. La cáscara de ñame y de yuca fueron obtenidas en empresas de alimentos en la ciudad de Cartagena, estas fueron lavadas tres veces usando agua destilada y secadas inicialmente por 24 horas en horno a una temperatura de $90^{\circ} \mathrm{C}$, posteriormente fueron reducidas de tamaño y tamizadas, seleccionando el tamaño de partícula del rango de 0,5-1 mm para la modificación química, pues en previas experimentaciones se determinó como el óptimo para el proceso de adsorción. Ambas biomasas fueron tratadas químicamente con ácido cítrico 0,6 M agitando $40 \mathrm{~g}$ de biomasa con 200mL de la solución de ácido cítrico a $200 \mathrm{rpm}$ a una temperatura constante de $60^{\circ} \mathrm{C}$ por 2 horas. Luego del tiempo de contacto, los bioadsorbentes fueron filtrados y lavados con agua destilada para retirar restos de ácido, y secados a $55^{\circ} \mathrm{C}$ por 24 horas (Altun y Pehlivan, 2012; Pitsariet al., 2013). Cabe destacar que esta modificación se basó en un mecanismo de carboxilación, en el cual el objetivo fue adicionar grupos carboxilos a la fibra tratada mediante la formación de un anhidro entre dos grupos carboxílicos debido a la perdida de una molécula de agua durante el calentamiento del ácido cítrico y la biomasa, que luego reacciona con un grupo hidroxil de la biomasa para formar un éster, y así aumentar la posibilidad de enlace con el ion metálico (Pitsariet al., 2013), tal como se observa en la Figura 1.

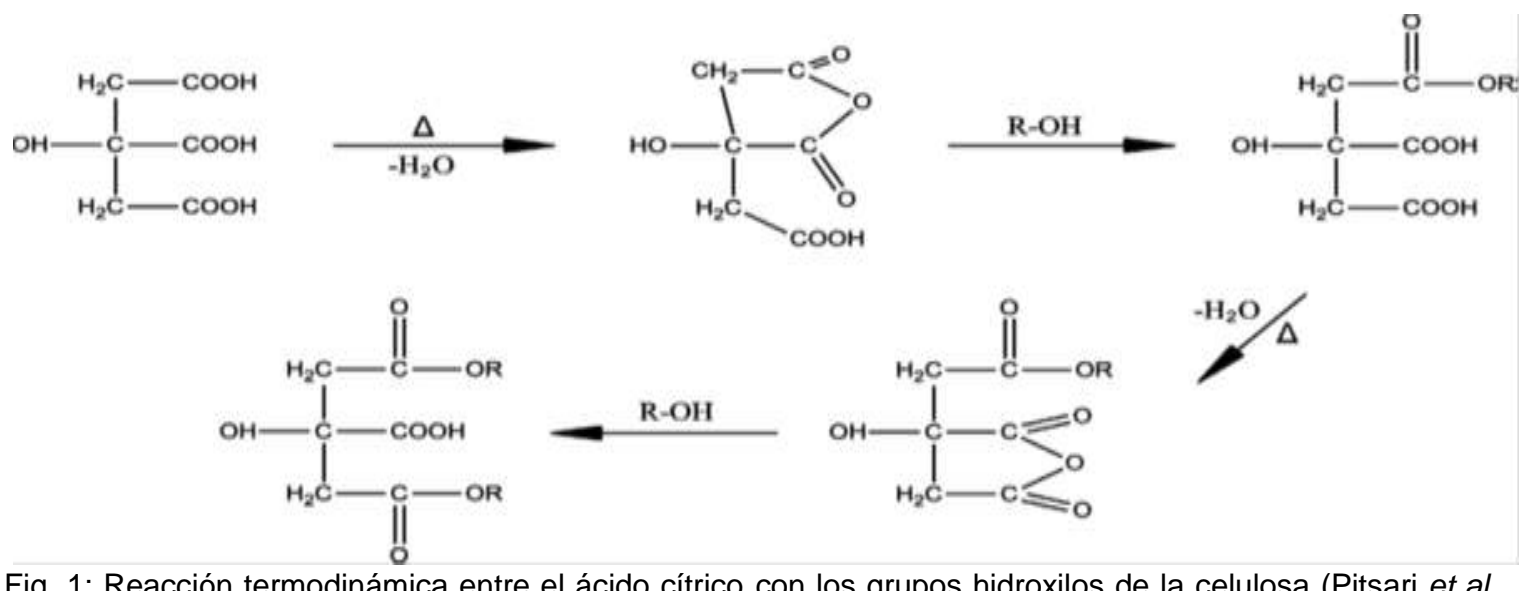

Fig. 1: Reacción termodinámica entre el ácido cítrico con los grupos hidroxilos de la celulosa (Pitsari et al., 2013 citado en Tejada C. et al., 2015)

\section{Caracterización fisicoquímica}

Una vez preparado los materiales adsorbentes se procedió a la caracterización físico-química de los mismos. Cabe destacar que características de superficie o texturales de las biomasas usadas no fueron determinadas en el presente trabajo. La caracterización realizada consistió en determinar los elementos y compuestos orgánicos presentes en estas por diferentes métodos analíticos tal como se muestra en la Tabla 1, todos los análisis fueron realizados en el laboratorio de Química Analítica de la Universidad de Cartagena.

Se realizó también un análisis por espectroscopía infrarroja por transformada de Fourier o FTIR por sus siglas en inglés (Fourier transform infrared spectroscopy) a cada una de las biomasas antes y después del proceso de remoción metálica para identificar los grupos funcionales que intervienen en la adsorción, para esto los adsorbentes fueron secados a $50^{\circ} \mathrm{C}$ por 24 horas y luego encapsulados en pellets de $\mathrm{KBr}$, para ser analizados en espectrofotómetro en el rango $4000-400 \mathrm{~cm}^{-1}$. 
Tabla 1: Pruebas de caracterización realizadas a los materiales adsorbentes.

\begin{tabular}{|c|c|}
\hline Muestra & Método utilizado \\
\hline Carbono (\%) & AOAC 949.14 \\
\hline Hidrogeno (\%) & AOAC 949.14 \\
\hline Nitrógeno (\%) & AOAC 984.13 KJELDAHL \\
\hline Azufre (ppm) & Digestión - nefelometría \\
\hline Cenizas (\%) & Termogravimetría \\
\hline Pectina (\%) & Digestión acida- termogravimetría \\
\hline Lignina (\%) & Fotocolorimetría \\
\hline Celulosa (\%) & Digestión - termogravimetría \\
\hline Hemicelulosa (\%) & Digestión - termogravimetría \\
\hline
\end{tabular}

\section{Pruebas de adsorción}

La solución patrón de 1000 ppm de $\mathrm{Pb}(\mathrm{II})$ fue preparada usando $\mathrm{Pb}\left(\mathrm{NO}_{3}\right)_{2}$ de grado analítico, diluyendo hasta llevar a la concentración necesaria. Inicialmente se realizó el estudio cinético usando una concentración inicial del metal de 100ppm. Las pruebas fueron realizadas a pH de 6 y éste fue ajustado usando $\mathrm{HCl}$ y $\mathrm{NaOH} 1 \mathrm{M}$, según lo reportado por Maldonado et al., (2011). Se midió la concentración del metal sobrenadante cada 30 minutos hasta lograr un equilibrio. La concentración final de plomo fue determinada por espectroscopia de absorción atómica, usando un equipo iCE 3000. El ajuste de los modelos cinéticos a los datos experimentales se realizó con los modelos mostrados en la Tabla 2.

Además, bajo las mejores condiciones de operación, evaluadas en estudios preliminares en la línea de investigación; fueron realizadas pruebas de adsorción con un diseño experimental tipo factorial para las dos biomasas en estudio, trabajando con un diseño $3^{2}$ donde se trabajó con dos variables independientes; el tamaño de partícula y el $\mathrm{pH}$, a tres niveles de variación; para el primero se probaron (0,355 , 0,5 y 1 mm), para el segundo se probaron valores de $\mathrm{pH}$ de $(2,4 \mathrm{y} 6)$, encontrando que el mejor valor para las dos biomasas en la adsorción de plomo fue a $\mathrm{pH}$ de 6; corroborando lo reportado por algunos autores (Maldonado et al., 2011). En el caso del tamaño de partícula se encontró que el mejor valor fue el de $1 \mathrm{~mm}$. Todos los experimentos fueron realizados a $25^{\circ} \mathrm{C}, 150 \mathrm{rpm}$ de velocidad de mezclado y tiempo de contacto 120min; se realizó el estudio a diferentes concentraciones iniciales de metal (25, 50, 75 y 100ppm) para determinar la capacidad máxima de adsorción de cada biomasa. El ajuste se realizó con los modelos de Langmuir y Freundlich. El análisis de los datos obtenidos fue realizado con la herramienta Solver de Excel determinando el ajuste de los modelos de acuerdo a la suma de los errores al cuadrado.

Tabla 2: Ecuaciones de cinética e isotermas y parámetros (Rosales et al., 1996; Hernández, 2008).

\begin{tabular}{|c|c|c|}
\hline Modelo & Ecuación & Parámetro \\
\hline Langmuir & $q=q_{m} \frac{b C_{e q}}{\left(1+b * C_{e q}\right)}$ & $\begin{array}{l}\text { C: concentración del adsorbato en el equilibrio (en mg/L), qm: } \\
\text { la capacidad máxima de adsorción (en } \mathrm{mg} / \mathrm{g}) \\
\text { b: constante de Langmuir de afinidad }(\mathrm{L} / \mathrm{mg})\end{array}$ \\
\hline Freundlich & $q=k_{f} * C^{1 / n}$ & $\begin{array}{l}\text { kf: constante de capacidad de adsorción } \\
\mathrm{n} \text { : constante de intensidad de adsorción }\end{array}$ \\
\hline Pseudo primer orden & $\frac{d q_{t}}{d t}=k_{a d}\left(q_{e}-q_{t}\right)$ & $\begin{array}{l}\text { qe: cantidades adsorbidas en el equilibrio } \\
\text { qt: cantidades adsorbidas en cualquier instante de tiempo } \\
(\mathrm{mg} / \mathrm{g}) \\
\mathrm{k}_{\text {ads }} \text { es la constante de adsorción del modelo }\left(\min ^{-1}\right) \text {. }\end{array}$ \\
\hline Pseudo segundo orden & $q_{t}=\frac{t}{\frac{1}{k_{2} * q_{e}^{2}}+\frac{t}{q_{e}}}$ & $\begin{array}{l}\text { k: constante cinética de Pseudo-segundo orden ( } / \mathrm{mmol} . \mathrm{min}) \\
\left.h=k_{2} * q_{e}^{2} \text {, siendo } \mathrm{h} \text { ( } \mathrm{mmol} / \mathrm{g} \cdot \mathrm{min}\right) \text { la velocidad inicial de } \\
\text { adsorción. }\end{array}$ \\
\hline Elovich & $q_{t}=\frac{1}{b} \ln (t)+\frac{1}{b} \ln (a * b)$ & $\begin{array}{l}q_{t} \text { cantidad de metal extraído en el tiempo t } \\
\text { a: es la velocidad inicial de adsorción } \\
\text { b: está relacionada con la superficie cubierta y la energía de } \\
\text { activación por quimisorción. }\end{array}$ \\
\hline Difusión intraparticular & $\log q_{t}=\log k_{\text {int }}+0,5 \log t$ & $\begin{array}{l}\text { qt: es la cantidad de metal adsorbido, t el tiempo de contacto } \\
\mathrm{k}_{\text {int: }} \text { coeficiente de difusión intraparticular }\end{array}$ \\
\hline
\end{tabular}

\section{RESULTADOS Y ANÁLISIS}

\section{Caracterización fisicoquímica de las cascaras utilizadas como bioadsorbentes}

Con el fin de conocer la composición del material adsorbente, se estudió el contenido de los distintos elementos presentes en la cáscara de yuca y la cáscara de ñame mediante un análisis elemental, determinándose el porcentaje de elementos y biopolímeros contenidos en la biomasa. Los resultados obtenidos se encuentran registrados en la Tabla 3. Para ambos biomateriales se observa que el carbono 
representa el compuesto con mayor porcentaje, confirmando la presencia de polisacáridos que representan un papel fundamental en la adsorción debido a su estructura, que cuenta con la presencia de grupos tales como alcoholes, ácidos, hidróxidos fenólicos, aldehídos y éteres que están relacionados con la capacidad de intercambio iónico de la biomasa por ser compuestos polares (Hernández, 2008). Asimismo, se observa que en las cáscaras de ñame hay mayor presencia de lignina que en la cáscara de yuca lo cual podría favorecer el proceso de adsorción en esta biomasa.

Tabla 3: Análisis elemental de los bioadsorbentes usados

\begin{tabular}{|l|c|c|}
\hline Muestra & Cáscara de yuca & Cáscara de ñame \\
\hline Carbono(\%) & 36,96 & 48,14 \\
\hline Hidrogeno (\%) & 3,98 & 5,44 \\
\hline Nitrógeno (\%) & 0,26 & 0,18 \\
\hline Azufre(ppm) & 0,12 & 0,08 \\
\hline Cenizas (\%) & 1,58 & 4,85 \\
\hline Pectina (\%) & 2,84 & 10,98 \\
\hline Lignina (\%) & 2,20 & 27,73 \\
\hline Celulosa (\%) & 18,47 & 13,08 \\
\hline Hemicelulosa (\%) & 6,01 & 6,47 \\
\hline
\end{tabular}

\section{Cinética de adsorción}

En las Figuras de la 2 a la 5, se muestra la cinética de adsorción de $\mathrm{Pb}$ (II) y el ajuste de los modelos cinéticos a los datos experimentales. De la cinética del proceso se establece que es la cáscara de ñame modificada con ácido cítrico el biomaterial que alcanza el equilibrio de manera más rápida, pues en los primeros 10 minutos del proceso ya se había logrado un $96 \%$ de remoción metálica a diferencia de un $70 \%$ con la cáscara de yuca. Esta situación podría ser explicada debido a la mayor presencia de lignina en la cáscara de ñame lo que aumenta el número de grupos funcionales hidroxilos y carboxílicos que favorecen el proceso de carboxilación con el ácido cítrico creando más sitios disponibles para la adsorción lo que a su vez aumentaría la posibilidad de retención del metal.

Al realizar el ajuste de los modelos cinéticos y obtener los parámetros mostrados en la Tabla 4, se establece que es el modelo cinético de Elovich el que describe de mejor forma el proceso de adsorción, ya que el valor de la suma de los errores al cuadrado así lo indica. Esto es un muestra de que el mecanismo controlante de la adsorción de $\mathrm{Pb}$ (II) sobre los biomateriales usados es una reacción de segundo orden, y además muestra que la superficie catalítica de los materiales es heterogénea, exhibiendo diferentes energías de activación, cabe aclarar que en este modelo, $\alpha$, la velocidad inicial de adsorción, se relaciona con la energía de quimisorción y $\beta$ con la superficie cubierta, lo que muestra que el metal es tomado más rápidamente por la cáscara de ñame, siendo este mismo material el que mayor superficie disponible presenta para el proceso de adsorción.

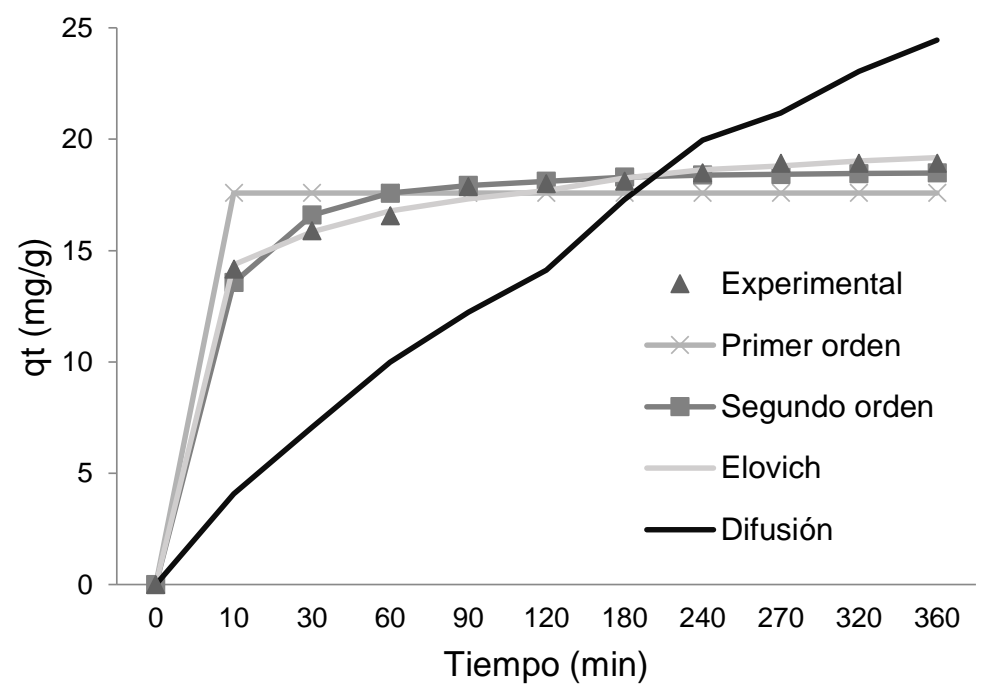

Fig. 2: Ajuste de modelos cinéticos a la adsorción de Pb (II) usando cáscara de yuca 


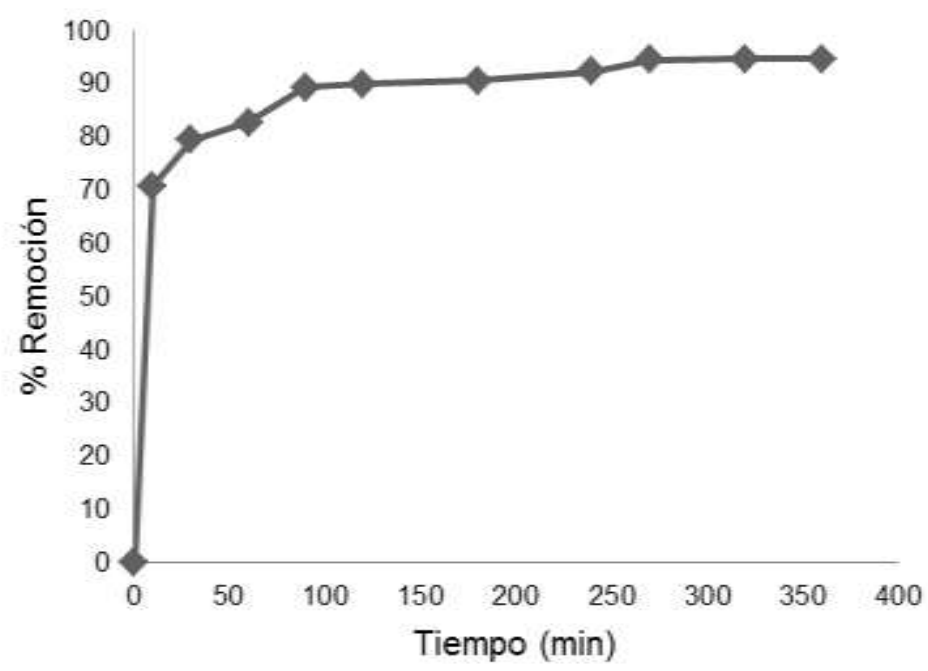

Fig. 3: Cinética de adsorción de Pb (II) usando cáscara de yuca

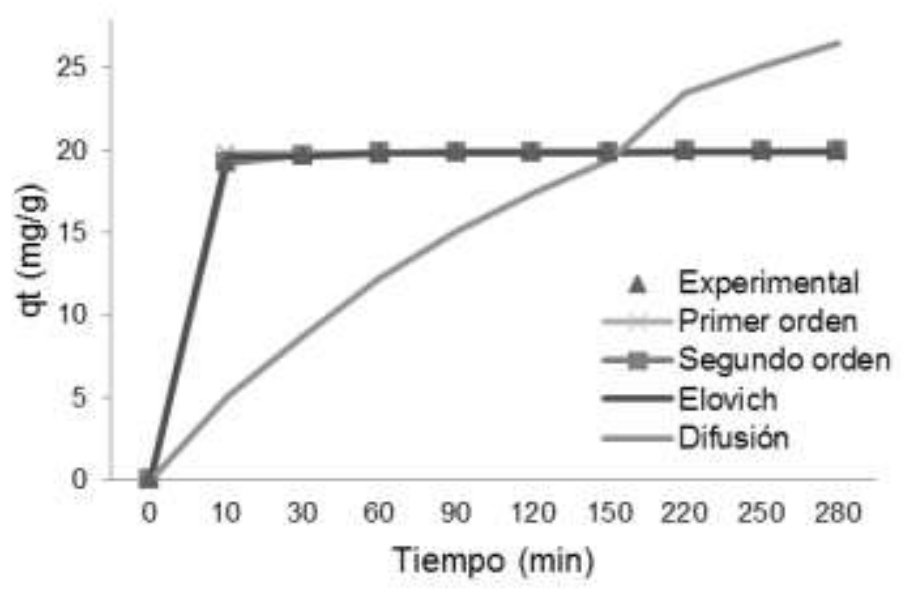

Fig. 4: Ajuste de modelos cinéticos a la adsorción de Pb (II) usando cáscara de ñame

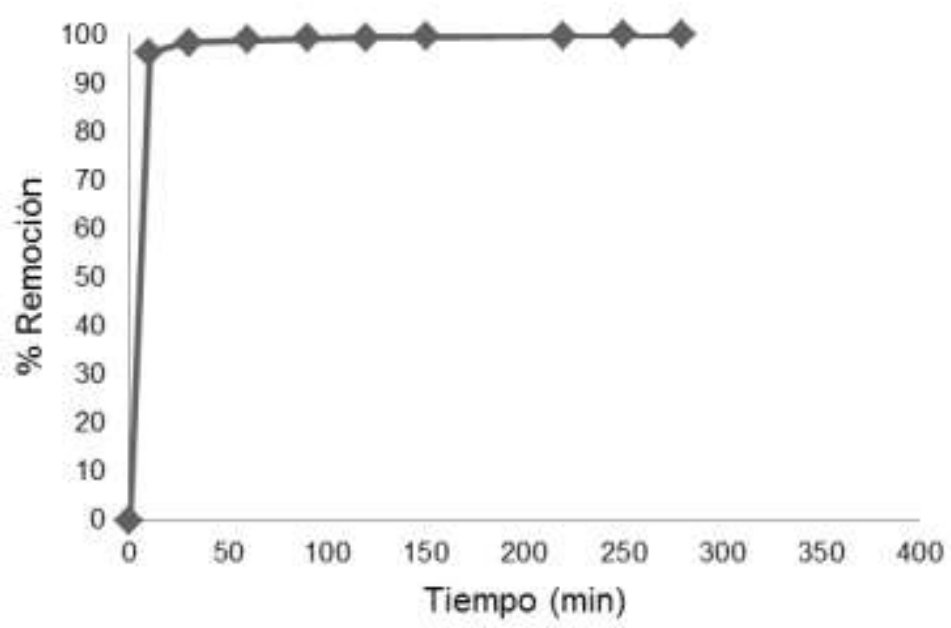

Fig. 5. Cinética de adsorción de Pb (II) usando cáscara de ñame

El efecto de la concentración inicial de $\mathrm{Pb}$ (II) fue estudiado con las isotermas de Freundlich y Langmuir, tal como se muestra en las Figuras 6 y 7 , y en la Tabla 5. Se encuentra la alta correlación de los datos experimentales con los modelos de Langmuir y Freundlich por lo cual estos pueden explicar la adsorción de ambos materiales satisfactoriamente. En cuanto a los parámetros calculados, b de la isoterma de Langmuir es un indicativo de la energía de enlace entre el metal y el ion metálico, por lo tanto se deduce que la cáscaras de ñame presentan una mayor energía de enlace que la cáscara de yuca, mientras que el valor de $1 / \mathrm{n}$, que en ambos casos es menor a 1, indica que la isoterma de Freundlich se puede aproximar a la de Langmuir. Así se establece que el enlace se da principalmente por fuerzas físicas, limitándose a la 
formación de una monocapa, además que el número de especies adsorbidas no excede el total de sitios disponibles y no se considera ninguna reacción de desorción que pueda tener lugar durante el proceso de remoción (Alves y Gil, 2009).

Tabla 4: Ajuste de modelos cinéticos a los datos experimentales de adsorción.

\begin{tabular}{|c|c|c|c|}
\hline Modelo cinético & Parámetros & Cáscara de yuca & Cáscara de ñame \\
\hline Primer orden & $q_{\mathrm{e} 1}(\mathrm{mg} / \mathrm{g})$ & 17,58 & 19,82 \\
\cline { 2 - 4 } & $\mathrm{k}_{1}\left(\mathrm{~min}^{-1}\right)$ & 20,70 & 1,53 \\
\cline { 2 - 4 } & Suma & 22,44 & 0,40 \\
\hline \multirow{4}{*}{ Segundo Orden } & $\mathrm{k}_{2}\left(\mathrm{~g} / \mathrm{mg}^{*} \mathrm{~min}\right)$ & 0,01 & 0,13 \\
\cline { 2 - 4 } & $\mathrm{q}_{\mathrm{e} 2}(\mathrm{mg} / \mathrm{g})$ & 18,68 & 19,97 \\
\cline { 2 - 4 } & Suma & 2,61 & 0,01 \\
\hline \multirow{7}{*}{ Elovich } & $\beta\left(\mathrm{g}^{\star} \mathrm{min} / \mathrm{mg}\right)$ & 0,74 & 11,78 \\
\cline { 2 - 4 } & $\alpha(\mathrm{mg} / \mathrm{g})$ & 5798,50 & $2,47 \mathrm{E}+98$ \\
\cline { 2 - 4 } & Suma & 0,62 & 0,15 \\
\hline \multirow{2}{*}{ Difusión } & $\mathrm{k}_{3}$ & 1,29 & 1,58 \\
\cline { 2 - 4 } & Suma & 325,25 & 491,75 \\
\hline
\end{tabular}

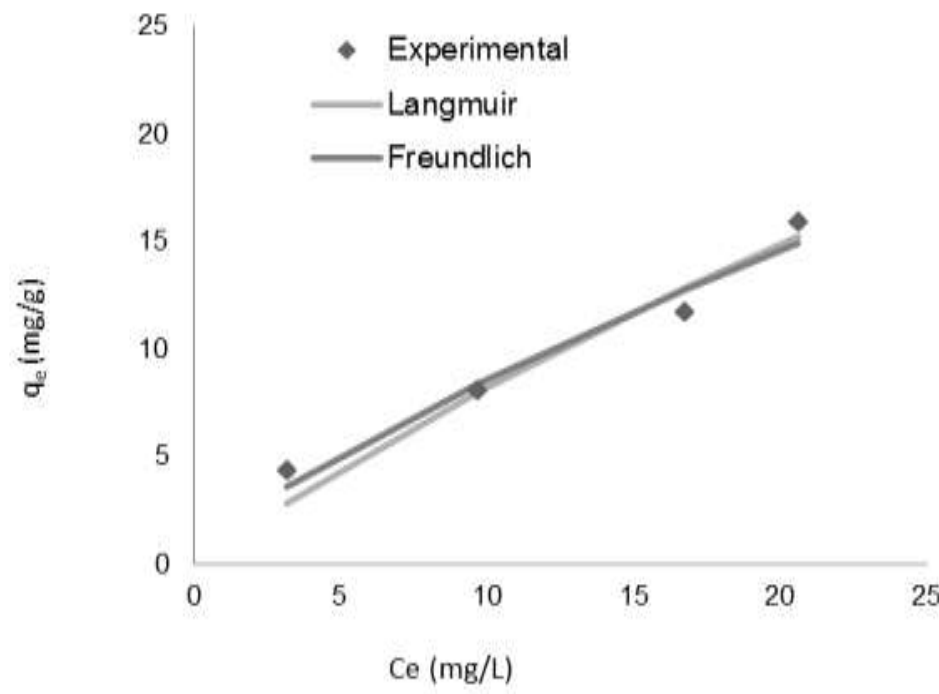

Fig. 6: Ajuste de Isotermas a la adsorción de Pb (II) usando cáscara de yuca

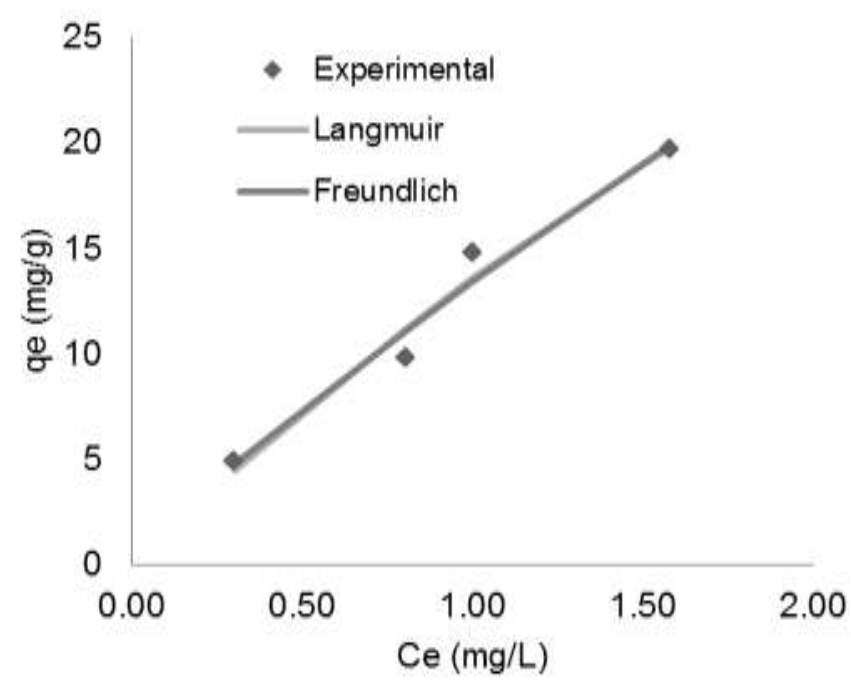

Fig. 7: Ajuste de Isotermas a la adsorción de Pb (II) usando cáscara de ñame. 
Tabla 5: Ajuste de isotermas de adsorción a los datos experimentales

\begin{tabular}{|l|c|c|c|}
\hline Modelo Isoterma & Parámetros & Cáscara de yuca & Cáscara de ñame \\
\hline \multirow{3}{*}{ Langmuir } & $q_{\max }(\mathrm{mg} / \mathrm{g})$ & 52,34 & 98,36 \\
\cline { 2 - 4 } & $\mathrm{b}(1 / \mathrm{mg})$ & 0,02 & 0,16 \\
\cline { 2 - 4 } & Suma & 4,03 & 3,69 \\
\hline Freundlich & $\mathrm{K}_{\mathrm{f}}(\mathrm{mg} / \mathrm{g})$ & 1,48 & 13,42 \\
\cline { 2 - 4 } & $1 / \mathrm{n}$ & 0,76 & 0,86 \\
\cline { 2 - 4 } & Suma & 2,80 & 3,70 \\
\hline
\end{tabular}

De acuerdo a la isoterma de Langmuir se encuentra que la cáscara de ñame modificada con ácido cítrico presenta una mayor capacidad de adsorción que la cáscara de yuca, lo cual puede ser relacionado con la presencia de lignina en el material sin modificación. Ahora si se comparan las capacidades de adsorción con los mismos materiales sin modificación trabajados por los autores en trabajos previos, se observa un incremento en la capacidad máxima de adsorción con la yuca, pues se obtuvo $11,5 \mathrm{mg} / \mathrm{g}$, mientras que con el ñame la remoción fue más exitosa sin modificación ya que se había alcanzado una capacidad de $362 \mathrm{mg} / \mathrm{g}$, por tanto se recomienda estudiar más el mecanismo de reacción de este material con el ácido cítrico. Si se compara con otros biomateriales que han sido usados para la remoción de $\mathrm{Pb}$ (II) como el aserrín modificado con $\mathrm{H}_{3} \mathrm{PO}_{4}$ 80,64mg/g (Ghasemiet al.,2014), avellana 128,18mg/g (Yuet al.,2013), almendra 8,08mg/g (Pehlivanet al.,2009), cáscara de banano 2,18mg/g (Anwar et al.,2010), y cáscara de naranja sulfurada $248,628 \mathrm{mg} / \mathrm{g}$ (Lianget al., 2011) todos estos trabajos son reportados a temperatura ambiente $\left(25-30^{\circ} \mathrm{C}\right)$, se establece que los materiales usados en el presente estudio presentan un alto potencial para la remoción de metales en solución acuosa.

En las Figuras 8 y 9, se muestran los FTIR correspondientes a la cáscara de yuca modificada con ácido cítrico, antes y después del proceso de adsorción. Al compararlos se encuentra la participación de los $\mathrm{O}-\mathrm{H}$ de los grupos poliméricos del biomaterial, puesto que se observa el cambio en la banda que aparece entre $3640-3310 \mathrm{~cm}^{-1}$, además se muestra el cambio en la banda de $3000-2900 \mathrm{~cm}^{-1}$, por lo que establece la participación de los grupos $\mathrm{C}-\mathrm{H}$. Así mismo, de los grupos $\mathrm{COO}$ y $\mathrm{C}=\mathrm{O}$ que aparecen entre 1740$1725 \mathrm{~cm}^{-1}$; en el análisis del material después de la adsorción aparecen nuevos picos entre $1300-1500 \mathrm{~cm}^{-1}$ que corresponden a la vibración $\mathrm{C}=\mathrm{C}$. Por último, se observan cambios en el espectro en la banda de 1150$700 \mathrm{~cm}^{-1}$ que muestran la contribución de los grupos $\mathrm{C}-\mathrm{O}-\mathrm{C}, \mathrm{C}-\mathrm{O}-\mathrm{P}$ y O-H de los polisacáridos (Li et al., 2008).

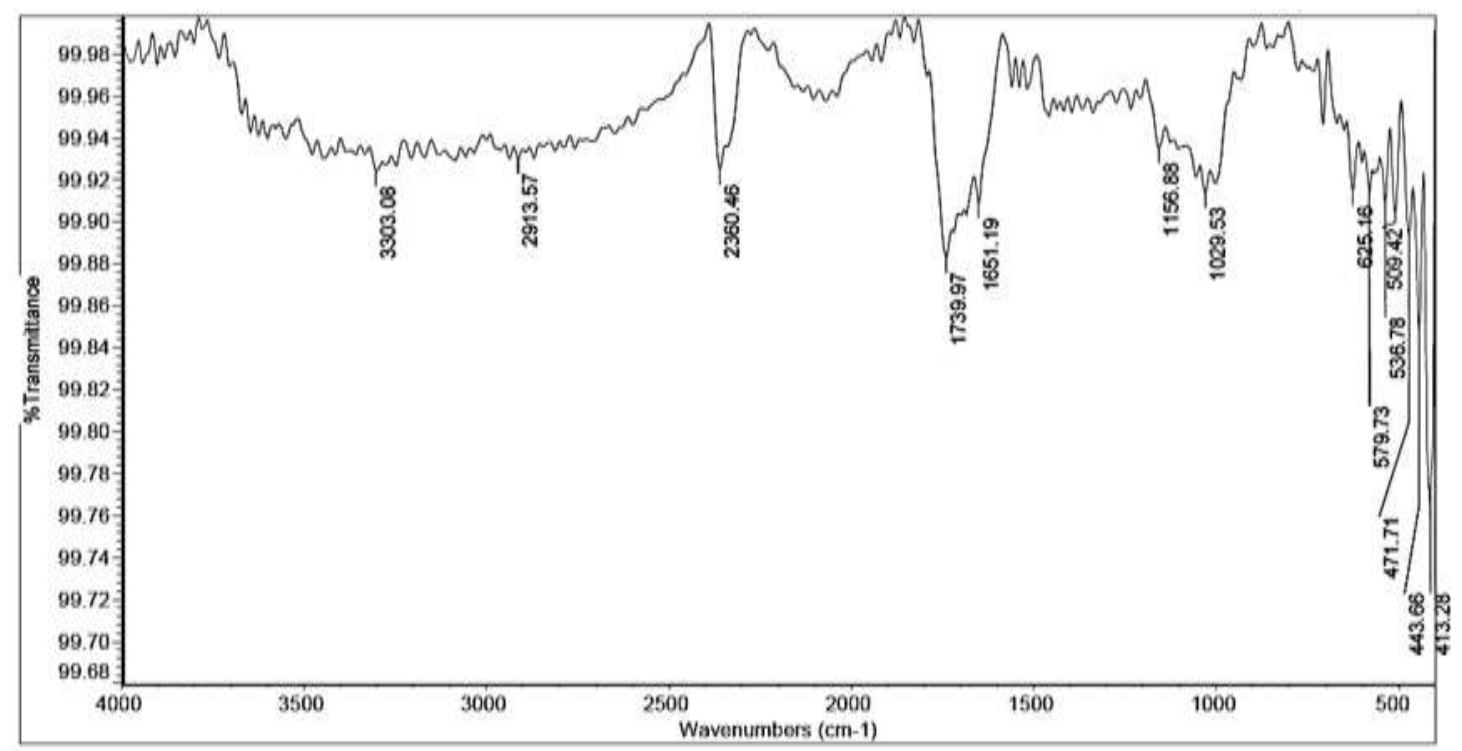

Fig. 8: Análisis FTIR de la cáscara de yuca modificada con ácido cítrico antes de la adsorción de $\mathrm{Pb}(\mathrm{II})$.

En las Figuras 10 y 11 se muestran los FTIR correspondientes a la cáscara de ñame modificada con ácido cítrico, antes y después del proceso de adsorción. Al compararlos se encuentra que no hay participación de los grupos $\mathrm{O}-\mathrm{H}$ pertenecientes a los grupos poliméricos de la cáscara de ñame, puesto que se observa un cambio casi que insignificante en la banda que aparece inicialmente a $3275 \mathrm{~cm}-1$ y luego aparece a $3280 \mathrm{~cm}^{-}$ 1, quizás esta pueda ser la razón por la cual hubo una disminución de la adsorción respecto a la biomasa sin modificar, pues estos grupos no fueron modificados, así se recomienda evaluar el mecanismo de reacción 
en un próximo estudio, donde quizás pueda ser usado un agente que permita la adición del metal a este grupo funcional, no obstante si es observado un cambio en la banda que aparece a $1200 \mathrm{~cm}^{-1}$, confirmando la participación del grupo funcional C-H en la adsorción de plomo, aparece un nuevo pico entre 1300-1500 $\mathrm{cm}-1$ que corresponde a la vibración $\mathrm{C}=\mathrm{C}$ (Li et al., 2008; Fisalet al.,2012).

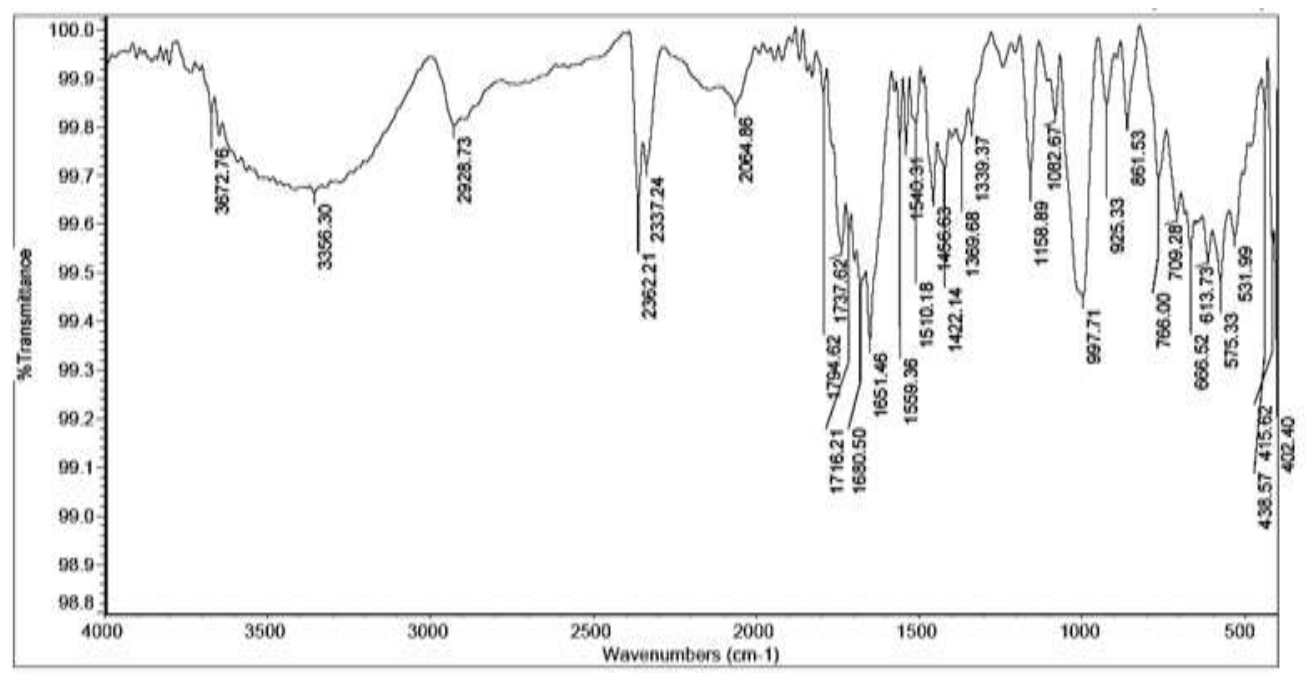

Fig. 9: Análisis FTIR de la cáscara de yuca modificada con ácido cítrico después de la adsorción de Pb(II).

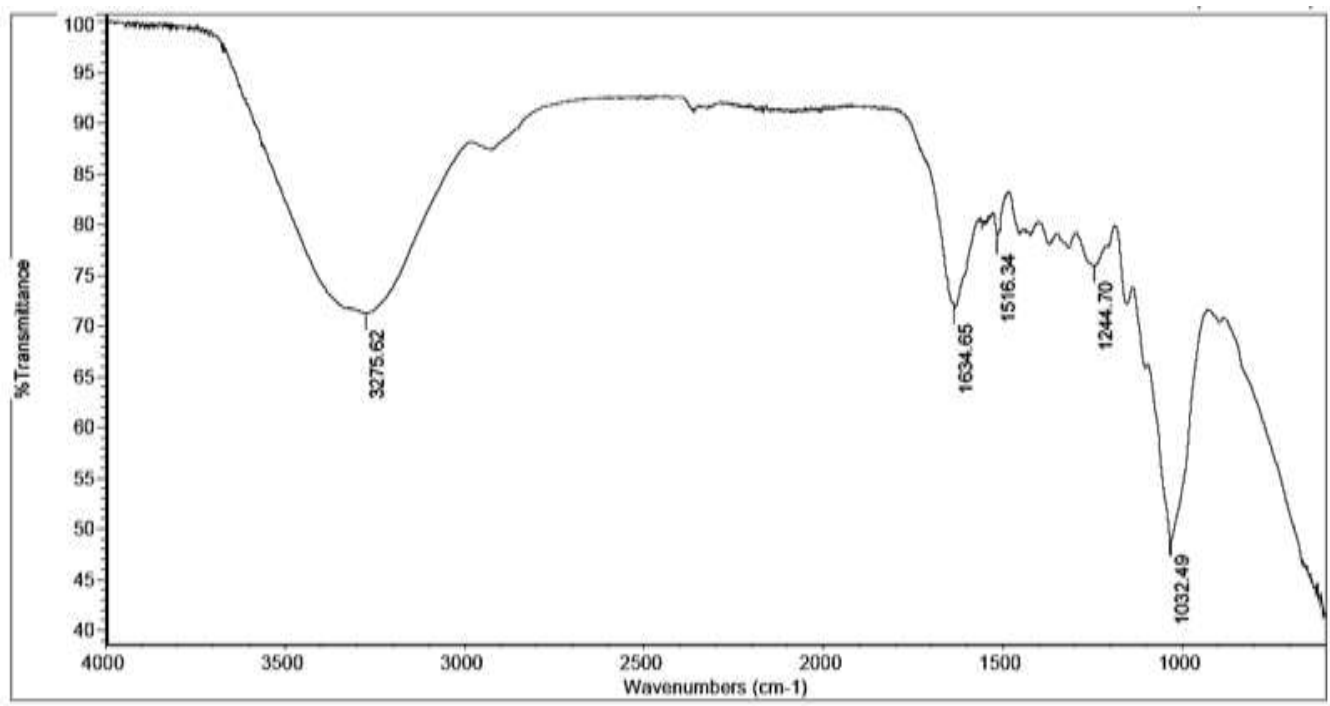

Fig. 10: Análisis FTIR de la cáscara de ñame modificada con ácido cítrico antes de la adsorción de Pb(II).

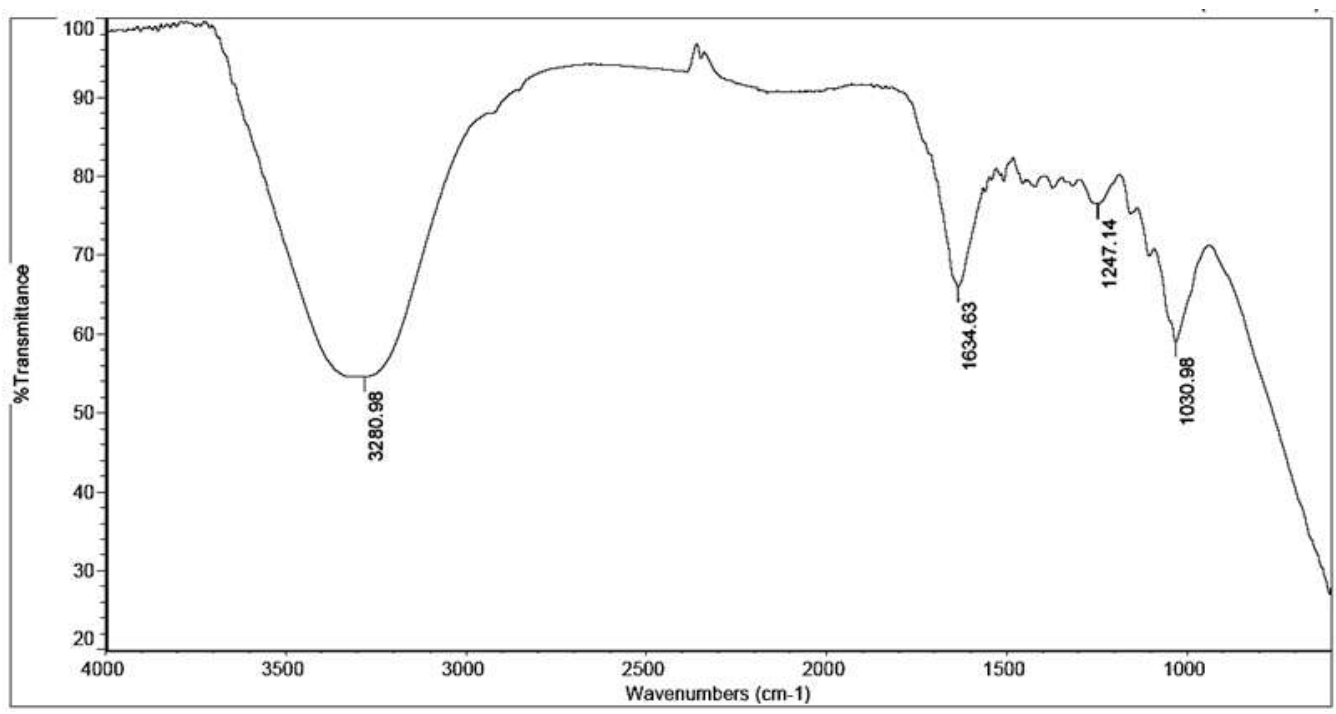

Fig. 11: Análisis FTIR de la cáscara de ñame modificada con ácido cítrico después de la adsorción de Pb(II). 


\section{CONCLUSIONES}

Se concluye que es posible el uso de materiales residuales de la agroindustria propia de la región caribe colombiana para la adsorción de plomo en solución acuosa, así se establece un valor agregado a lo que actualmente es considerado un desecho, y que además se convierte en un problema para el ambiente por su acumulación.

Se obtuvieron altas capacidades de adsorción de $\mathrm{Pb}(\mathrm{II})$ para los biomateriales trabajados, haciendo uso del mecanismo de carboxilación con ácido cítrico, capacidades comparables con otros adsorbentes, pero en el caso de las cáscaras de ñame desmejoran los resultados obtenidos con la biomasa sin modificar. Lo anterior puede ser relacionado con la no participación de los grupos hidroxilo de la misma, por lo cual se recomienda un estudio del mecanismo de reacción que permita hacer uso de un agente enlazante.

Se encuentra que el modelo de Elovich se ajusta a los datos experimentales lo que permite establecer que la superficie de los biomateriales es heterogénea. Asimismo, de acuerdo al ajuste de la isoterma de Langmuir a los datos trabajados, se concluye que el enlace se da principalmente por fuerzas físicas, limitándose a la formación de una monocapa, donde el número de especies adsorbidas no excede el total de sitios disponibles y no se considera ninguna reacción de desorción que pueda tener lugar durante el proceso de remoción. Del análisis FTIR de los materiales se encuentra que en general son los grupos hidroxilo, metileno y éter los principales involucrados en la adsorción de $\mathrm{Pb}$ (II).

\section{REFERENCIAS}

Altun, T. y Pehlivan, E. Removal of $\mathrm{Cr}(\mathrm{VI})$ from aqueous solutions by modified walnut shells, Food Chemistry, 132, 693-700 (2012)

Alves, L.V. y Gil, L.F. Adsorption of $\mathrm{Cu}(\mathrm{II}), \mathrm{Cd}(\mathrm{II})$ and $\mathrm{Pb}(\mathrm{II})$ from aqueous single metal solutions by succinylated twice-mercerized sugarcane bagasse functionalized with triethylenetetramine, Water research, 43, 4479-4488 (2009)

Anwar, J., Shafique, U., Waheed-uz-Zaman, Salman, M., Dar, A., y Anwar, S. Removal of Pb (II) and Cd (II) from water by adsorption on peels of banana, Bioresource Technology, 101, 1752-1755 (2010)

Brousse, M., Vergara, M., Zubreski, E., Cruz, N. y Martos, M. Cinética de adsorción de agua en purés deshidratados de mandioca (Manihot esculenta Crantz) (Water adsorption kinetics in dehydrated mashed cassava (Manihot esculenta Crantz)), Revista Venezolana de Ciencia y Tecnología de Alimentos, 22, 53-57 (2012)

Edoun, M. y Kuitche, A. Sorption isotherms and isosteric energy of Manihot esculenta leaves. International Food Research Journal 21(1), 343-348 (2014)

Fisal, A., Wan, D., Mohd, A. y Rosinah, R. Cocoa (Theobroma cacao) shell-based activated carbon by CO2 activation in removing of Cationic dye from aqueous solution: Kinetics and equilibrium studies, Chemical engineering research and design, 90, 1480-1490 (2012)

Ghasemi, M., Mu, N., Ghasemi, N. y Khosravi-fard, Y. A novel agricultural waste based adsorbent for the removal of $\mathrm{Pb}$ (II) from aqueous solution: Kinetics, equilibrium and thermodynamic studies, Journal of Industrial and Engineering Chemistry, 20, 454-461 (2014)

Hernández, A. Influencia del tamaño de partícula en la biosorción de plomo con raspo de uva, Tesis de Maestría, Escola Tècnica Superior d'Enginyeria Industrial de Barcelona, Universitat Politècnica de Catalunya. Departament d'Enginyeria Química, Barcelona, España (2008)

Jun-Xia, Y., Li-Yan, W., Ru-An, C., Yue-Fei, Z., Zhi-Gao, X., Guo, J. Competitive adsorption of Pb2+ and $\mathrm{Cd} 2+$ on magnetic modified sugarcane bagasse prepared by two simple steps. Applied Surface Science, 268, $163-170(2013)$

Kosasih, A., Febrianto, J., Sunarso, J., Ju, Y.H., Indraswati, N. y Ismadji, S. Sequestering of Cu(II) from aqueous solution using cassava peel (Manihot esculenta). Journal of hazardous materials. Journal of Hazardous Materials, 180, 366-374 (2010) 
Li, X., Tang, Y., Cao, X., Lu, D., Luo, F. Y Shao, W. Preparation and evaluation of orange peel cellulose adsorbents for effective removal of cadmium, zinc, cobalt and nickel. Colloids and Surfaces A: Physicochem, Eng. Aspects, 317, 512-521 (2008)

Maldonado, A., Luque, C. y Urquizo, D. Biosorción de plomo de aguas contaminadas utilizando pennisetum clandestinum hochst (kikuyo). Rev. LatinAm. Metal. Mat., S4: 52-57 (2012)

Ning-chuan, F. y Xue-yi, G. Characterization of adsorptive capacity and mechanisms on adsorption of copper, lead and zinc by modified orange peel. Transactions Nonferrous Metals Society of China, 22:1224-31 (2012)

Onwu, F., Abii, T. A. y Ogbonna, A. Kinetic studies on the use of agricultural wastes for the removal of lead, cadmium and nickel ions from aqueous solutions. Journal of Chemical and Pharmaceutical Research, $6(12): 471-480(2014)$

Owamah, H., Chukwujindul, L. y Asiagwu, A. Biosorptive capacity of yam peels waste for the removal of dye from aqueous solutions. Civil and Environmental Research. Vol 3 (1): 36-47 (2013)

Pehlivan, E., Altun, T., Cetin, S. y lqbal, M. Lead sorption by waste biomass of hazelnut and almond shell, Journal of Hazardous Materials, 167, 1203-1208 (2009)

Pitsari, S., Tsoufakis, E. y Loizidou, M. Enhanced lead adsorption by unbleached newspaper pulp modified, Chemical Engineering Journal, 223, 18-30 (2013)

Rosales, J. y Paucar, R. Uso de la cáscara de yuca en raciones para cerdos en crecimiento.Folia amazónica, 8(2), 105-118 (1996)

Shih-Wei, L., Chun-I, L. y Li-Hwa, W. Kinetic study on lead (II) ion removal by adsorption onto peanut hull ash, Journal of the Taiwan Institute of Chemical Engineers, 42, 166-172 (2011)

Tejada C., Villabona, A. y Ruiz, E. Cinética de adsorción de $\mathrm{Cr}(\mathrm{VI})$ usando biomasas residuales modifcadas químicamente en sistemas por lotes y continuo. Revista ION, 28 (1), 29-41 (2015)

Vinoth, M., Lim, H.Y., Xavier, R., Marimuthu, K., Sreeramanan, S., Mas, H. y Kathiresan, S. Removal of methyl orange from solutions using yam leaf fibers. International Journal of Chem Tech Research, 2 (4), 1892-1900 (2010)

Vizcaíno, L. y Fuentes, N. Biosorción de $\mathrm{Cd}, \mathrm{Pb}$ y $\mathrm{Zn}$ por biomasa pretratada de algas rojas, cáscara de naranja y tuna. Ciencia Ingeniería Neogranadina, 25(1): 43-60 (2015) 
